\title{
Mol-D a Database and a Web Service within the Serbian Virtual Observatory and the Virtual Atomic and Molecular Data Centre
}

\author{
Vladimir A. Srećković ${ }^{1}$, Darko Jevremović ${ }^{2}$, Veljko Vujčićéc, \\ Ljubinko M. Ignjatović ${ }^{1}$, Nenad Milovanović ${ }^{2}$, Sanja Erkapić ${ }^{2}$ \\ and Milan S. Dimitrijević ${ }^{2,4,5}$ \\ ${ }^{1}$ Institute of Physics Belgrade, University of Belgrade, \\ P.O. Box 57, 11001, Belgrade, Serbia \\ email: vlada@ipb.ac.rs \\ ${ }^{2}$ Astronomical Observatory, Volgina 7, 11060 Belgrade, Serbia \\ email: darko@aob.rs \\ ${ }^{3}$ Faculty of Organizational Sciences, Univesity of Belgrade, Serbia \\ ${ }^{4}$ Observatoire de Paris, 92195 Meudon Cedex, France \\ email: mdimitrijevic@aob.rs \\ ${ }^{5}$ IHIS Techno experts, Batajnički put 23, 11080 Zemun, Serbia
}

\begin{abstract}
In this contribution we report the current stage of the MOLecular Dissociation (MOL-D) database which is a web service within the Serbian virtual observatory (SerVO) and node within Virtual Atomic and Molecular Data Center (VAMDC). MOL-D is an atomic and molecular (A\&M) database devoted to the modelling of stellar atmospheres, laboratory plasmas, industrial plasmas etc. The initial stage of development was done at the end of 2014, when the service for data connected with hydrogen and helium molecular ions was done. In the next stage of the development of MOL-D, we include new cross-sections and rate coefficients for processes which involve species such as $X \mathrm{H}^{+}$, where $X$ is atom of some metal. Data are important for the exploring of the interstellar medium as well as for the early Universe chemistry and for the modeling of stellar and solar atmospheres. In this poster, we present our ongoing work and plans for the future.
\end{abstract}

Keywords. Virtual Observatory, Astroinformatics, Stars, Atomic and Molecular Data, Databases

\section{Introduction}

In the era of large sky surveys and numerous large telescopes, data volumes have grown exponentially and increased from terabytes into tens or hundreds of petabytes (Brunner et al. 2001, Djorgovski et al. 2013, York et al. 2000) and will increased even faster (LSST Science Collaboration et al. 2009). At the same time, simultaneously a huge amount of information and data (produced by powerful supercomputer simulations) are distributed through the global network infrastructure and stored in the networks of archives using new technologies (Allock et al. 2002, Tate et al. 2016). Consequently, the status of data-oriented science, research methods, algorithms, and techniques become very important. Operative processing and scientific exploitation of such large data sets remains as one of the key motivations behind the astronomical Virtual Observatory and a new emerging discipline Astroinformatics (Borne 2013, Brescia \& Longo 2013). This has led to integration of computer science, physics, statistics, astrophysics etc. In this contribution, we give an overview of the motivations, current stage, and technological principles of MOL-D database within Virtual Observatories. 


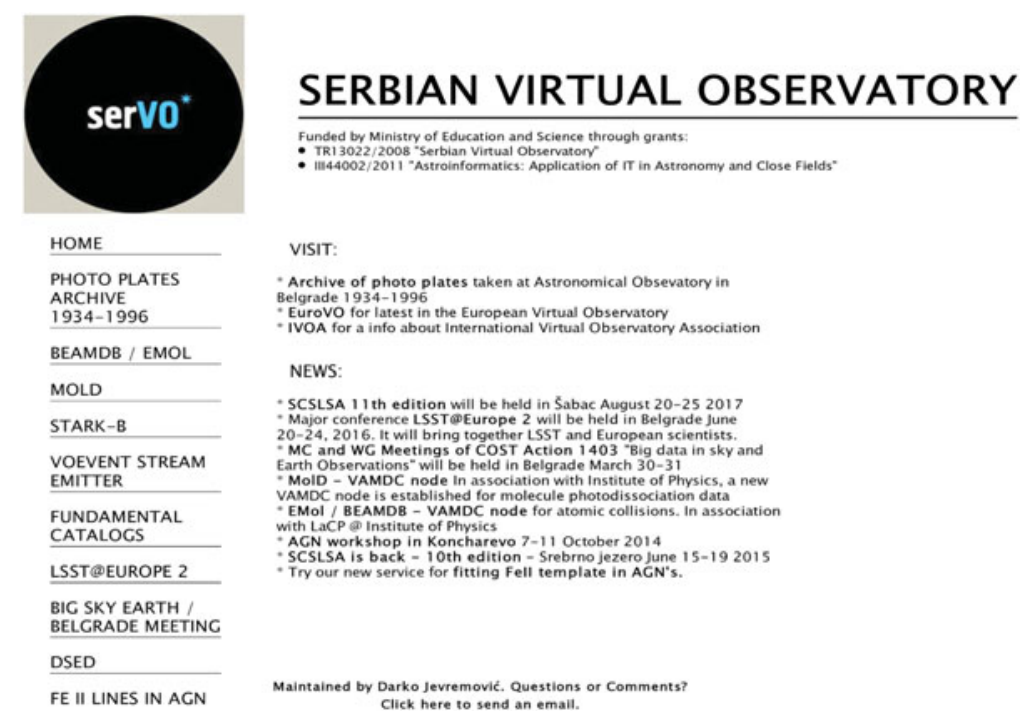

Figure 1. The home page of the SerVO (Jevremović et al. 2009).

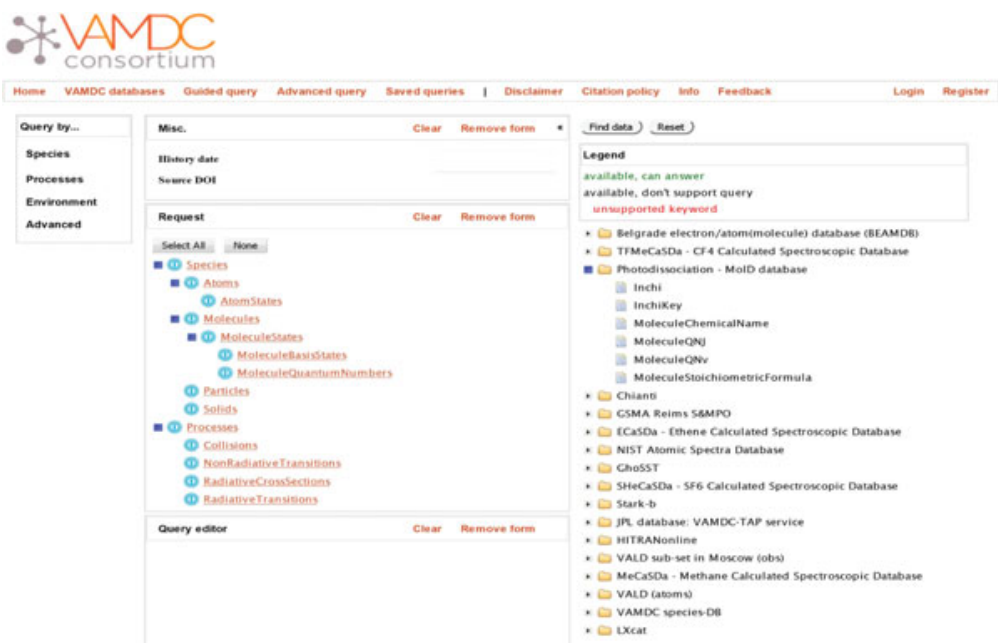

Figure 2. VAMDC (Dubernet et al. 2016) portal snapshot (http://www.portal.vamdc.eu).

\section{Overview of MOL-D}

Many fields in astronomy, atmospheric physics, chemistry, industry etc. depend on data for A\&M interaction processes. A reliable exchange of such data has become crucial. In this context, we are developing the MOL-D database. MOL-D database is a collection of cross-sections and rate coefficients for specific collisional processes (Vujčić et al. 2015). It can be accessed via http://servo.aob.rs/mold/ of Serbian Virtual Observatory (SerVO, see Fig.1) or accessed as a web service http://portal.vamdc.eu/ which is part of the Virtual Atomic and Molecular Data Center (VAMDC, see Fig.2).

The database contains photodissociation cross-sections for the individual ro-vibrational states of the diatomic molecular ions and rate coefficients for the atom-Rydberg atom chemi-ionization and inverse electron-ion-atom chemi-recombination processes. A graphical interface is provided at 'Homepage' (Fig.3). The web interface offers access to data for 


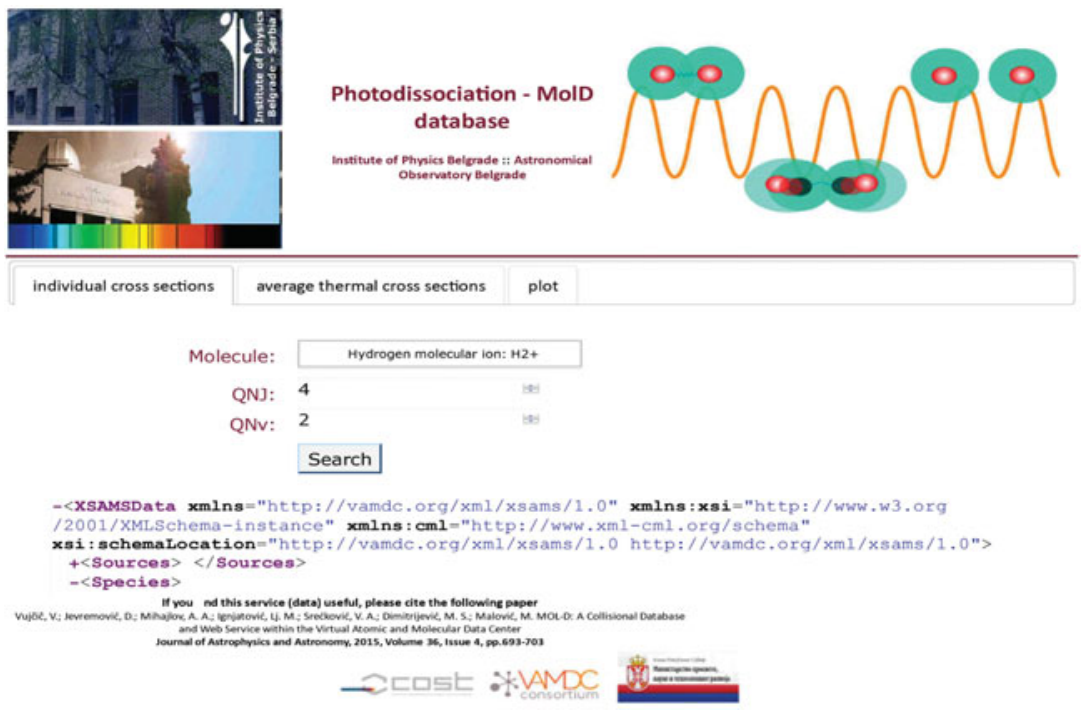

Figure 3. Homepage of MOL-D. Access to the data: the graphical interface. Data set is represented in XSAMS (Extensible Markup Language (XML) Schema for Atoms, Molecules and Solids) format.

photodissociation (bound-free) cross-sections of hydrogen $\mathrm{H}_{2}^{+}$and helium $\mathrm{He}_{2}^{+}$molecular ions (see e.g. figure 3 ) as well as the corresponding averaged thermal photodissociation cross-sections for the requested wavelength and temperature. All data sets are linked to the original article so that it is fully citable.

\subsection{Level 2 Release}

At the end of 2016 MOL-D is in the stage 2 of development. We are including new crosssections and rate coefficients data for processes which involve species such as $\mathrm{MgH}^{+}$, $\mathrm{HeH}^{+}, \mathrm{LiH}^{+}, \mathrm{NaH}^{+}, \mathrm{SiH}^{+}$which are important for exploring of the interstellar medium, the early Universe chemistry and for the modeling of different stellar and solar atmospheres (Srećković et al. 2014, Coppola et al. 2016, Ignjatović et al. 2014a, Ignjatović et al. 2014b, Vujčić et al. 2015). Our plans are incremental inclusion of data from our papers concerning atomic and molecular processes important for modeling different stellar atmospheres and laboratory plasmas as they become published. Along with database updates, we intend to develop new web utilities and interfaces for SerVO MOL-D website.

\section{Database technical description}

VAMDC is an international consortium which has built a well documented, secure, flexible interoperable e-science platform permitting an automated exchange of atomic and molecular data. VAMDC e-infrastructure defines protocols for retrieving remote data as well as format for representing these data. The ultimate goal is interoperability of the data along various distributed nodes. Access to the MOL-D data is possible via Table Access Protocol (TAP), a Virtual Observatory standard of a web service or via AJAX (Asynchronous JavaScript and XML)-enabled web interface (http://servo.aob.rs/mold). Both queries return data in XSAMS (XML Schema for Atoms, Molecules and Solids) format (Braams et al. 2016). The XSAMS schema provides a framework for a structured presentation of atomic, molecular, and particle-solid-interaction data in an XML file. Underlying application is written in Django, a Python web framework and represents a customization and extension of VAMDC's NodeSoftware. Additional on-site utilities include: data selection based on molecule name and QNJ/QNv numbers; average thermal 
cross section calculation based on temperature for a specific molecule and wavelength; plotting average thermal cross sections along available wavelengths for a given temperature.

\section{Concluding remarks}

Atomic and molecular databases in today's science have become essential for modeling and interpretation of data provided by observations and laboratory measurements (Srećković et al. 2014, Mihajlov et al. 2012, Mihajlov et al. 2013, Marinković et al. 2015). Nowadays, very important resources of such data are dissipated, presented in different, non-standardized ways, available through a variety of highly specialized and often not very well documented interfaces. As a consequence the full exploitation and interconnection of all available data is limited and the free exchange of these data requires the definition of some standards and tools that help users and producers in this process. The continuation of such developments and services is crucial. This is the purpose of further development of MOL-D database within SerVO and VAMDC.

\section{Acknowledgements}

This work was supported by the Ministry of Education, Science and Technological Development of the Republic of Serbia Grants III44002 (Astroinformatics: Application of IT in Astronomy and Close Fields) and OI176002 (Influence of collisional processes on the astrophysical plasma spectra). A part of this work has been supported by VAMDC.

\section{References}

Allcock, B., et al. 2002, Parallel Comput., 120, 28.5: 749-771

Borne, K. 2013, Virtual observatories, data mining and astroinformatics, in Planets, Stars and Stellar Systems, vol. 2, pp. 404-443, Wiley, 2013.

Braams, B. J., et al. 2016, International XML Schema for Atoms, Molecules and Solids (XSAMS) Version 1.0, Report INDC (NDS)-0706 (Vienna: International Atomic Energy Agency)

Brescia, M. \& Longo, G. 2013, Nucl Instrum Methods Phys Res A, 720, 92

Brunner, R., Djorgovski, S. G., Prince, T., \& Szalay, A. 2001, Massive Datasets in Astronomy, in Handbook of Massive Data Sets (J. Abello et al., eds.), Boston: Kluwer, 931.

Coppola, C. M., Mizzi, G., Bruno, D., et al. 2016, Mon. Not. R. Astron. Soc, 457, 3732

Djorgovski, S. G., Mahabal, A. A., Drake, A. J., Graham, M. J., \& Donalek, C. 2013, Sky surveys, in Planets, Stars and Stellar Systems. Astronomical Techniques, Software, and Data (T. Oswalt and H. Bond, eds.), vol. 2, pp. 223-281, Wiley, 2013.

Dubernet, M. L., Antony, B. K., Ba, Y. A., et al. 2016, J.Phys.B, 49, 074003

Ignjatović, L. M., Mihajlov, A. A., Srećković, V. A., \& Dimitrijević, M. S. 2014a, Mon. Not. R. Astron. Soc, 439, 2342

Ignjatović, L. M., Mihajlov, A. A., Srećković, V. A., \& Dimitrijević, M. S. 2014b, Mon. Not. R. Astron. Soc, 441, 1504

Jevremović, D., Dimitrijević, M. S., Popović, L. Č., et al. 2009, New Astron. Revs, 53, 222

LSST Science Collaboration, Abell, P. A., Allison, J., et al. 2009, arXiv:0912.0201

Marinković, B. P., Vujčić, V., Sushko, G., et al. 2015, Nucl.Instrum.Methods B, 354, 90

Mihajlov, A. A., Srećković, V. A., Ignjatović, L. J. M., Dimitrijević, M. S., \& Metropoulos, A. 2012, J Phys Conf Ser, 397, 012054

Mihajlov, A. A., Ignjatović, L. M., Srećković, V. A., Dimitrijević, M. S., \& Metropoulos, A. 2013, Mon. Not. R. Astron. Soc, 431, 589

Srećković, V. A., Mihajlov, A. A., Ignjatović, L. M., \& Dimitrijević, M. S. 2014, Adv. Space Res., 54, 1264

Tate, J., et al. 2016, in Introduction to storage area networks, IBM Redbooks.

Vujčič, V., Jevremović, D., Mihajlov, A. A., Ignjatović, Lj. M., Srećković, V. A., Dimitrijević, M. S., \& Malović, M. 2015, J. Astrophys. Astron., 36, 693

York, D. G., Adelman, J., Anderson, J. E., Jr., et al. 2000, AJ, 120, 1579 